R. Htstória, São Paulo, D. 129-131, p. 141-152, ago.-dez./93 a ago.-dez./94.

\title{
JUIFS ET PAÏENS DANS LE MONDE GRECO-ROMAIN
}

\author{
Mireille Hadas-Lebel *
}

\begin{abstract}
RESUMO: $O$ anti-semitismo existiu na sociednde pagá antes de desenvolver-se no seio do cristianismo? Os juleus, cujo monotelsmo os isolava do meio ambiente, constituiram frequentemente minorias ameaģadas entre os paghos; no entanto - e apesar de suas revoltas contra Roma - seu culto recebeu a proteçäo das leis romanas att o começo do imperio cristâa. $\mathbf{A}$ imagem do judeu na literatum greco-romana que aparece a partir do III século está bem longe de ser uaiformemente negativa. No I século, é preciso distinguir a irritaçăo dos romanos tradicionais diante do proselitismo judeu e as calúnias dos Alexandrinos suscitadas pela pretençăo dos judeus a cidadania. Apesar do conflito religioso fundumental, nâo há nada da obsessłło criach mais tarde pelo odio teológico.
\end{abstract}

PALAVRAS-CHAVE: Antiguidade Greco-Romana, judalsmo, racismo, anti-semitismo, religiåo.

L'élude des relations entre Juifs et païens dans l'Antiquité a souvent été abordée sous l'angle de l'antisémitisme. De quand date l'antisémitisme? Peut-on dire à propos des quelques textes grecs ou latins qui présentent les Juifs sous un jour défavorable, "qu'il n'est presque pas une note de la cacophonie médiévale et moderne qui ne soit perceptible dans le choeur des écrivains anciens"(S. W. Baron)? Ou doit-on convenir avec Jules Isaac que l'antisémitisme a des racines chrétiennes? Telle est la problématique qui domine, pour des raisons aisément compréhensibles, depuis le lendemain de la seconde guerre mondiale. Elle a été développée dans d'importants ouvrages auxquels nous ne pouvons que renvoyer.

L'usage même du terme d' "antisémitisme" est-il approprié s'agissant de l'Antiquité? Ce terme, surgi en Allemagne en 1879, suppose l'apparition de la notion de "sémitisme" et donc la théorie des races, extrapolée au 19e siècle à partir des découvertes de la philologie. Tout en étant parfaitement conscients de l'anachronisme que constitue l'usage de ce terme, d'aucuns l'appliquent néamoins aux périodes antérieurs au $19 \mathrm{e}$ siècle, considérant que

- Institut National des Langues et Civilisations Orientales - Paris

1 Voir notamment les ouvrages de R. RUETHER, V. NIKIPROWETZKY, J. GAGER cite dans la bibliographie. 
HADAS-LEBEL, Miseilte. Juils el païens dans le monde gréco-romain.

la chose a existé bien avant le mot. Le terme d" anlijudaïsmen", que d'aulres voudraient lui substituer en toule rigueur, est parfois réservé au connit théologique qui oppose christianisme el judaĩsme ${ }^{2}$, à l'exclusion de toute dimension économique, sociale, politique ou raciale, bien que. la haine théologique ait inévitablement des conséquences sur ces divers plans.

Cet article, venant après tant d'éludes sur le sujet, se propose d'examiner le regard des nations sur Israēl. Dans la variété des altitudes observées, y en a-t-il q̨ui puissent se comparer à l'antisémitisme moderne?

\section{Minorilés menacées}

Le caractère prétendument éternel d'un antisémilisme qui seraił "aussi vieux que le judaïsme meme" est souvent mis en relation avec le monothéisme. C'est parce qu' lsraël place sa foi en un Dicu unique qu'il se sépare des nations. Encore ! ii faut-il beaucoup de temps pour affermir sa croyance: loute l'histoire des Hébreux depuis la sortic d'Egypte jusqu'à la captivité de Babylone peut çtre lue comme la lente et chaolique progression de l'idée d'un Dieu immatériel chez un peuple qui n'aspire qu'à adorer les divinités de ses voisins.

Après la destruction du premier Temple (-586), le relour d'exil el la restauration du Sanctuaire confortent la foi des déscendants des Judéens exilés - c'est-à-dire des Juirs (du latin Judaeus). Désormais le monothéisme est fortement ancré dans leur conscience et ils s'efforcent de se protéger de la contagion idolâtre sur le plan des moeurs. C'esı ainsi qu'lsraël devienı "un peuple qui demeure à part" (Nombres XXIII,9). Ce particularisme ne manque pas d'etre vivement ressenti par leurs voisins, surtout là où les Juifs vivent en minoritaires parmi d'autres nations. L'antagonisme religieux, même s'il subsiste quelque temps sans violence, risque toujours de dégénérer en conflit, au déıriment de la minorité, plus vulnérable. .

La première note de ce qui ressemble à de l'antisémilisme moderne se fait entendre dans le livre d'Esther. Dans le vaste empire d'Assuérus qui ne compte pas moins de cent vingt sept provinces, les Juifs ne sont qu'un peuple parmi d'autres, avec "sa langue el son écriture" (III,12); mais leur religion leur interdit la prosternation devant les puissants de ce monde. Le conflit religieux tourne au politique; quand Aman dénonce au roi ce peuple "dispersén", qui vit à part dans les provinces du royaume, il insiste sur son 
R. Ilıslórla, Sāo Paulo, n. 129-131, p. 141-152, ago.-dez./93 a ago.-dez./94.

insoumission: "ils n'observent pas les lois du roi" (1II,8). Lal violence de la haine causée par un événemenl isole (le refus de Mardochée de se prosterner devant Aman), la généralisation de celte haine à toul une catégorie d'individus et la volonte d'extermination qui s'ensuil, donne des arguments aux tenants de l'exislence d'un antisémilisme aussi vieux que le judaïsme lui-même. Cependant, it ne faul pas oublier que le livre d'Esıher est un écrit juif, que les paroles mises dans la bouche d'Aman sont imaginaires, qu'il n'existe aucune trace historique de ce qui, aus yeux des Juifs, est le récil d'un miracle. Il est probable que les exilés de Judée dispersés dans l'empire perse aeent çà et là rencontré quelque hoslilité, comme toule minorité aux coutumes spécifiques, mais la protection des autorités a toujours joué.

Sans remonter jusqu'au récit de l'Exode, l'Égypte est un pays où une minorité juive toujours ménacée, disparait el se reconstitue périodiquement. Des archives du 5ème siècle retrouvées sur le site d'Eléphantine (Assouan) permettent de retracer la vie d'une garnison juive installée là par le pouvoir perse $^{3}$. Après une ère de relative sécurité, le temple juif, qui y avait été élevé, esı delruit par la populace à l'instigation des prêtres égyptiens: dans un pays où le culte animal était répandu, le sacrifice d'agneaux à l'occasion de Páque ne pouvait apparaître que comme un sacrilège. Le conflit religieux est ici le détonateur mais il se superpose à un conflit politiquẹ l'hostilité envers le pouvoir perse et ses agents.

Dans l'Égypte ptolémaïque où ils furent généralement heureux, les Juifs connurent également une alerte. Selon une légende vivace parmi eux, le roi Plolénée IV Philométor $(-221-204)^{4}$ - ou plus vraisemblablement Plolémée Physcon (-145-116) ${ }^{5}$ - pour se venger du général juif Onias qui, lors d'une querelle dynastique, ne l'avait pas soutenu, réunit tous les Juifs, femmes et enfants compris, et les livra ligolés à un "troupeau d'éléphants préalablement énivrés. Or, ceux-ci, au lieu de les piétiner, se précipitèrent sur leurs ennemis. Une fêle spéciale était cếcébréc dans la communauté d'Alexandrie en commémoration de ce miracle. Ici, la source de la persécution esı donc plutoı presentée comme politique.

Cependanı, encore au débul du XIer siècle C.E., le conflit entre le monothéisme juif et lá zoolâtrie égyptienne alimente l'hostilité réciproque.

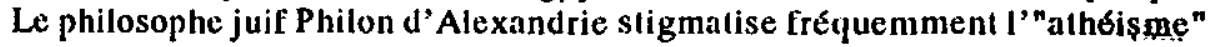

\footnotetext{
3 C.f. J. MELEZE, Les Jifs $d E_{g y p h}$, ip. $37 \mathrm{ct}$ sy, et pp. 115.116.

4 Selon le livre III des Maccibes.

5 Selon Flavius Josèphe dans le Contre Apion I1, 51-55. MELEZE, J. (op.cit., p. 121) estime que "la concision de Plavius Jusèphe confère à sa version une crélibilité que ne semble pas mériter le pathus romantique di llIéme livre des Maccabées".
} 
HADAS-LEBEL, Mireille. Juifs et païens Jans te monde gréco-romain.

égyplien car vénérer des bêtes nuisibles comme le crocodile n'est pas de la piété. Sur celte opposition religieuse se greffe un ressentiment social lorsque les indigènes égypticns voient les Juifs se ranger du côté du pouvoir grec puis romain dans leur pays, el ensuite lorsque les Grecs d'Egypte, désormais dominés par Rome, s'aperçoivent que ces Juifs devenus très hellénisés prétendent avoir droit de cité à Alexandrie.

En 38 éclate dans celte ville un vérilable pogrom qui est le résultat de toutes ces tensions accumulées. Les excès de la popülace sont encouragés par le préfet romain Flaccus qui espère rentrer en grâce auprès de Caligula, en sévissant contre les seuls habitants de l'empire qui refusent irréductiblement le culte impérial:

\section{La protection des lois:}

Loin d'ctre hostile aux Juifs qu'elie ne rencontre pas avant le -IIème siècle, Rome a signé en -161 un traité d'amitié et d'alliance avec Juda Maccabée qui menait la révolte contre la domination de la Syrie sélcucide. Les Juifs n'eurenı finalemen! pas besoin de l'aide romaine pour recouvrer leur indépendance. Ils continuèrent d'entretenir de bons rapports avec une Rome encore lointaine et le traité fut renouvelé périodiquement. Au moment de la grande expansion romaine en Méditerranée orientale, Pompée profita d'une querelle dynastique pour prendre Jérusalen (en -63) et confier la Judée au prétendant qu'il soutenait, Hyrcan II, entièrement manipulé par un Iduméen ${ }^{6}$, Antipater. Rome domine désormais indirectement le pays par l'intermediaire de ses hommes-liges, Antipater puis son fils Hérode qui prend le titre de roi en -40. Du moins ceux-ci savent-ils lui expliquer les susceptibilités religieuses de leur coreligionnaires de la diaspora. C'est ainst qu'en - 46 Jules César édicte une série de décrets protégeant le culte juif, confirmés plus tard par Auguste, el appliqués partout où il y a-des Juifs.

La diaspora juive esı dès celte époque considérable. Alors qu'il n'y a pas plus d'un million d'habitants (et vraisemblablement moins) en Judée même, on évalue à six ou sept millions le nombre d'habitants de l'empire romain (un sur huit ou un sur dix) qui se proclament Juifs ${ }^{7}$; parmi eux, un nombre croissant de prosélytes. Les décrets romains autorisent les Juifs à se

6 Les Iduméens, habilants des steppes du Sud, avaient eté convertis au judaïme par Jean Hyrcan en -129 .

7 JUSTER, J. Les Juifs dans I"empire romain. Paris, 1914. 
R. IIlstórin, Sîo Paulo, n. 129-131, p. 141-152, ago.-dez./93 a ago.-dez./94.

rcunir pour célébrer leurs fêtes ou à exporter leur contribution (un demi-sicle par mâle de plus de vingl ans) au Temple de Jérusalem alors que le droit de réunion el l'autorisation d'exportation d'argent sont strictement limilés par aillcurs; ${ }^{8}$; ils les dispensent du service militaire afin qu'ils n'aient pas à profaner le sabbat el les lois alimentaires, les relèvent de toule obligation à comparaître en justice le sabbal el les jours de fête, leur permeltent de recourir à leur propre juridiction pour leurs affaires ${ }^{9}$. C'est bien là la preuve que l'on veut ménager une minorité assez importante pour risquer de créer des troubles.

Quand, à la suite des exactions des procurateurs romains et dans une atmosphère d'effervescence messianique, éclate en + 66 la grande révolte des Juifs contre Rome, on assiste dans plusicurs cilés à des déferlements populaires anti-Juifs. Mais, à la veille de la révolie contre Rome, ce furent souvent les Juifs qui prirent l'initiative des hostilites, comme a Césarée ${ }^{10}$ ou à Scythopolis (Belh Shéan) et surtoul à Alexandrie où le préfet Tiberius Julius Alexander, un aposial, neveu de Philon d'Alexandrie, donna la troupe contre ses anciens coreligionnaires qui réagissaient aux provocations des Grecs, faisant parmi les Juifs, dî Flavius Josèphe, 50.000 morts (BJ 488-489).

Ces quelques cas se situent déjà dans un contexte de gucrre. Its ne doivent pas faire oublier les nombreuses cités de tout le bassin méditerranén, y compris Rome, où il ne se passa rien. Ils illusirent simplement la difficile coexistence de populations aux moeurs diverses, dans un contexle d'agitation politique. Quant aux aulorités romaines, elles voient les Juils comme un peuple sédilieux qui menace lá Pax Romana; les guerres menées contre eux relèvent des guerres coloniales.

Il haut souligner que malgré les deux révolles de la Judée, les lois romaines prolégeanı les Juifs ne furent jamais abrogées el que le cultc impérial ne leur lut jamais imposé. Les premières législations anti-juives commencent dans l'empire byzantin chrétien el sont motivées par des rivalités purement religieuses.

8 Cicéron tans le Prö Flacco défend le consul Flaccus yui avail confisqué les sommes envoyées par les Juifs de la province d'Asie.

9 Cf. JOSËPIE, Flavitus. Antiquites juives XIV, 228 a 240, Voir SAULNIER, Christiane. Wis romaines sur les Juils selon JOSĖPIIE, Flavius. Rentre Biblique, 88, 1981, pp 161-195.

10 Cr. JOSEPIIE De Bello Judaico II, 266. Qunnd ensuite les Césaréens massactèrent les Juirs de leur cité en représailles les Juifs dévastèrent cinq cilés de la Decapole et plusieurs autres lucalités ( $B J$ II, 457-460). Les cilés réagirent, mais certaines, comme Siubn, Apamé, Gernso, épargnèrent leurs Juits (BJ 479). A part celle d'Alcxandrie, les gramles communautes du bassin métitermnéen se furent ms inçuiétées. 
IIADAS-LEBEL, Mireille. Juifs el paīens dass le monde gréco-romain.

\section{L'image du Juif dans la littéralure gréco-latine}

Si pour le Juifs, Grecs et Romains rentrent dans la catégorie des idolatres, pour ces Jerniers les Juifs appartiennent à la catégorie des Barbares orientaux, envers lesquels ils éprouvent, à Jes rares exceptions près, un sentiment gênéral de supériorité. Il existe cependant, entre les Juifs et les autres Barbares, une différence nolable qui met du temps à se préciser dans l'esprit des Grecs el des Romains: c'est qu'ils n'acceptent pas à bras ouverts loùt l'hellénisme ou toute la romanité et gardent leur spécificité.

\section{Un peuple de philosophes?}

La première mention des Juifs apparaîr au -IVème siècle dans un fragment du trailé Sur la piété de Théophraste. Ils sonl présentés comme un "peuple de philosophes" qui s'entrelient de la divinilé el, la nuit, contemple les éloiles'. Il s'agil là d'une description du monothéisme, lequel pour les grees, "ne relève pas de la pratique religieuse, polythéiste par définilion, mais de la philosophic", comme le souligne très justement J. Mélèze ${ }^{12}$. Un peu plus tard, le voyageur Mégasthène compare les Juifs aux brahamanes indiens, aulres délenteurs d'une certaine sagesse barbare ${ }^{13}$. Au -Illème siècle, le péripatélicien Cléarque de Soles fäit descendre les Juifs des philosophes de l'Inde en s'appuyant sur un témoignage d'Aristole ${ }^{14}$. Vers le mềne temps, un certain Hermippe de Smyrne affirme que Pythagore a emprunté l'essentiel de sa doctrine aux Juifs ${ }^{15}$.

Dans tous ces fragments, les Juifs sont cités plutôt fugitivement par des auteurs Grecs qui, pour les besoins de leur démonstration, cultivent le thème de la sagesse orientale connue par ouï-dire. Il reste qu'ils les englobent parmi les détenteurs de cette sagesse.

Sans faire une place privilegiée aux Juifs, Epictète (X50-130 e.c.) met sur le même plan Juifs, Syriens, Égyptiens et Romains, et affirme qu'ils

11 C. REINACH Textes, p. 7-8; STERN I n. 4, pp. 8-12.

12 L'image du Juif dams la pensée grecque vers $300 \mathrm{~m}$ rant notre bre dans Greece and Rome in Enetz Israel, Jérusalem, 1990 , p. 107.

13 REINACH, p 15; STERN I n. 14, PP. 45-46.

14 REINACH, pp. 10-12 STERN I, n. 15, pp. 49-50. Ce témuignage est réproduit par Diogène Lnęrce an XIIIème siècle.

15 REINACI1, pp. 39-40; STERN I, n. 25,26, pp. 95-96. 
R. IIstúria, Stio Paulo, n. 129-131, p. 141-1.52, ago.-dez./93 a ago,-dez./94.

s'accordent tous pour tenter de parvenir à la sainteté, bien que les moyens préconisés diffèrent ${ }^{16}$.

\section{Un mode de vie d part}

L'absence d'images dans le culte juif retient l'attention de plusieurs auteurs à diverses époques. Varron (-116-27) qui identifie le Dieu des Juils à Jupiter, donne en example la pureté de leur culte sans images qu'il croit correspondre à la vieille religion romaine? ${ }^{17}$. Au XIllème siècle c.e., Dion Cassius constate: "ces hommes se dislinguenı du reste de l'humanité par tout leur genre de vie, pour ainsi dire, mais particulièrement en ce qu'ils n'honorent qucun des autres dieux et n'en adorent qu'un seul avec une grande ferveur" ${ }^{18}$. Mais, deux siècles plus tôt, Pline l'Ancien voyait dans une telle attilude une impiété caractérisée: "gens contumelia, numinum insignis" 19 .

La spécificité du mode de vie juif est signalée pour la première lois vers -300 par Hécatéc d'Abuèré ${ }^{20}$. Tout en présentant de manièré élogieuse l'oeuvre législatrice de Moïse, il note que elui-ci élablit des coutumes entièrement différents de celles des autres nations, et qu'"en souvenir de l'exil de son peuple, it institua un mode de vie insociable et hostile aux étrangers".

Cette accusation en sourdine est reprise à parlir du XIer siecle par des auteurs latins qui choisissent souvent le mode de la satire. Séneque ne comprend pas que l'on s'adonne "à la paresse" un jour sur sepl el que, ce jour-là, on n'éteigne pas les lampes à huile qui dégagent de la fumée ${ }^{21}$. Perse concentre ses traits satiriques contre l'observance du sabbat (Saturae V 176-184). Martial croit qu'il s'agit d'un jeône hebdomadaire (Epigrammata 1V,4). Pélrone (fragmeni 37) se demande si les Juifs n'adorent pas lé porc puisqu'ils réfusent de manger la viande de cel animal ${ }^{22}$ et les poètes satiriques de la fin du siècle se riènt de cette "clémence traditionnelle qui

16 STERN $\mathrm{t}, \mathrm{n} . \mathbf{2 5 3}$, p. 542.543.

17 REINACII, p. ; STERN 1, n. 72a-724; STERN, The Jents in Greek and Lutin Literature, p. 1125.

18 REINACI, ip. 182-183; STERN II, n. 406, pp. 349-350.

19 Histoire Naturelle XIII, 46 (STERN I, n. 214, p. 491).

20 REINACH, p. 15-20; STERN I, n. 11-12, Pp. 26-39.

21 STERN I, n. 186, p. 431.

22 Plutaryue discute de la question de savoir si les Juifs s'abstiennent de manger du porc par respect ou pror répulsion: Qunestiones Comriviales IV, 4, 4-6 (G69c-G72b) = STERN I, n. 258, P12. 550-552. 
HADAS-LEBEL, Mireille. Juifs et paiens dans le monde gréco-romain.

laisse les pourceaux mourir de vieillesse ${ }^{23}$. La circoncision est un inépuisable sujet de grossières plaisanteries ${ }^{24}$.

Le ton est netlement plus acerbe chez Tacite qui leur reproche de "manger à parı, dormir à part" et chercher à se distinguer en toul des autres hommes (Histoires V). Quintilien a une plurase sur "la nation pernicieuse aux autres" ${ }^{25}$. Au tournant du lllème siècle encore, Philostrate, reconstituant le dialogue du philosophe Apollonios de Tyane avec Vespasien, lui fait dire qu'il eat mieux valu ne pas conquérir ce peuple fait "d'hommes qui ont imaginé une vie insociable, qui ne partagent avec ses semblables ni la lable ni les libations, ni les prières, ni les sacrifices" ${ }^{26}$.

De meme, l'accusation de haine du genre humain es! lancée contre les Chrétiens puisqu'ils refusent, en raison de leurs convictions, de partager les coutumes et cérémonies des autres sujets de l'empire.

\section{Reaction au prostlytisme}

Quand, au premir sièclè, commence à s'exprimer l'agacement des Romains ou leur dérision, le judaīsme esı en pleine expansion. Comme le signale un siècle plus tard Dion Cassius, la dénomination de "loudaios" n'est pas limitée à un peuple, on l'étend à lous les hommes, même d'cthnie différente, qui suivent les lois des Juifs. Celte espèce se rencontre meme parmi les Romains; bien des fois réprimés, ils ont toujours repris des forces et ont fini par conquérir te droit de pratiquer libremente leurs usages (ibid.).

La plupart des réactions enregistrées ci-dessus proviennent donc en fail de l'irritation de vieux Romains face aux progrès qu'enregistraient les pratiques juives dans Rome meme $e^{27}$. La convertion se faisait souvent sur deux générations, comme l'indique Juvénal (Satires XIV): le père s'était contenté d'observer le sabbat et les lois alimentaires puis il avait fait circoncire son fils. Celui-ci, "élevé dans le mépris des lois romaines n'aprend, n'observe, ne révère que la loi judaïque, tout ce que Moïse a transmis à ses adeples dans un volume mystéricux" (vers 100-103). La fierlé romaine s'accomode mal de voir des Romains suivre les coutumes

JUVÉNAL, Satires VI, 160 .

MARTIAL, Epigrummata VI, 30,35,55,82; XI, 94; Hornce, Sermones, 1,9,60-78.

Intitutio Orratorio III,7,21.

REINACII, p 176, STERN II, n. 403, pp. 340-341.

C. HORACE, Sermones I,4,139-143. Plutarque, dans son inite Sur la Superstition (3, p. 166 A) mentionne Egalement le sabbat du nombre des coutumes étrangeres aduptes par les Grecs (STERN I, a. 255, p 549). 
K. II lstórta, Sĩo P’aulo, n. 129-131, p. 141-152, ago.-dez./93 a ago.-dez./94.

d'immigrés, de descendants d'altranchis traités avec mépris, dont on raille la pauvreléc menue monnaic, des vagabonds qui campent dans le bosquet de la nymphe Egéire $^{29}$ "Les vaincus imposenl leurs lois aux vainqueurs", gronde Sénèque ${ }^{30}$.

Ils sont lout naturellement l'objet d'une certaine xénophobie, à l'égal d'ailleurs des Égyptiens, des Syriens et d'autres orientaux qui afnuent à Rome. Sous Tibère, les cultes égyptiens avaient élé interdits et les Juifs avaient été chassés (en l'an X19) de Rome où ils revinrent d'ailleurs assez rapidement. Dès le régne de Claude, se sont plulôt les chrétiens qui crisłallisent l'animosité; cet empereur, nous dit Sútone, "chassa les Juifs qui se soulevaient sans cesse à l'instigation d'un certain Chrestos". Sous Néron, la pérseculion s'intensifia contre eux. Par suile, malgré les soulèvements locaux en Judée, c'est l'expansion du christianisme qui posa de problèmes aux autorités romaines. Dans l'empire byzantin, le dernier empereur païen, Julien dit "l'apostal", philosophe et chantre de l'hellénisme contre le christianisme, exprime sa préférence pour le judaīsme délenteur d'une longue Iradilion, face à une religion trop nouvelle encore pour prélendre à la légilimité.

\section{Les cas alexandrin}

Dans le monde gréco-romain, Alexandric constilue un cas à part. Les dissenssions inter-elhniques qui s'y élaient dévelopées sous les derniers Plolémées finirent par aboutir, comme on l'a vu, à des explosions populaires contre la minorité juive et finalement à l'anéantissement de celle-ci sous le règne de Trajan (entre XI15 ot X117).

La haine entre les Juifs et les Égypliens repose sur de Irès vieilles rivalilés politiques et religieuses. L'Exode s'en fail l'écho du colê juif el, du coté égyplien, un prêtre du nom Manćthon, écrivant en grec au -lllème sièclc, dont Flavius Josèphe a préservé quelques extraits dans le Contre Apion. Manéthon accuse les Hébreux de n'être à l'origine rien d'autre qu'un ramassis d'infirmes el de lépreux égypliens menés par un ancien prêtre d'Héliopolis du nom d'Osarseph yui pril le nom de Moïse et leur donna une constitution en contradiction absolue avec les coutumes locales. Plutot qu'une forme de racisme, celle accusation nous paraît être un type de

30 STERN I, n. 186, p. 4.31: Victi victoribus leges dederunt. 
IIADAS-LEBEL, Mireilte. Juifs et païens dnns le munde gréco-romain.

calomnie banal extrêmement répandu dans l'Orient ancien. Quiconque veut rabaisser un ennemi héréditaire lui allribue des origines infammantes. La Bible elle même en donne maint examples: les Cananéens sont les descendants de Cham, le moins estimable des fils de Noé, les Moabiles et les Ammonites sont nés d'un inceste et Ismaël est le fils d'une servante.

La particularité d'Alexandrie, c'est qu'au đébut du XIer siècle quand les relations entre Juifs et Grecs ou Égyptiens hellénisés d'Alexandrie s'envenimèrent notamment à propos du droit de cité réclamé par les Juifs, la fable des "impurs" fut reprise par divers polémisles comme Lysimaque, Cherémon et Apion afin de leur refuser ce droit.

Deux légendes calomnieuses, nées probablement en Syrie ${ }^{31}$ après la profanation du temple par Antiochus Epiphane en -167 ou peu après la prise de Jérusalem par Pompée en -63 , vinrent se greffer là-dessus. L'interdiction fajte aux élrangers de pénétrer dans le Temple élait sans doute à l'origine de toutes sortes de fantasmes sur ce qui pouvait bien s'y trouver. Si les historiens confirmèrent que Pompée ne vit aucune statue dans le Saint des Saints, l'imagination populaire ne se contenta pas de celte affirmation. Selon les rumeurs rapportées par Apio, on y aurait retrouvé une tête d'âne ou encore un captif grec engraissé en vue d'un sacrifice. La seconde fable, bien plus pernicieuse, constitue la première accusation de crime rituel contre les Juifs: utilisée ensuite par les païens contre les chrétiens, elle devait être retournée par ceux-ci contre les Juifs à partir du Moyen Age.

C'est aux auteurs alexandrins que pense S.W. Baron en écrivant "qu'il n'est pas une note de la cacophonie médiévale ou moderne qui nẹ soit perceptible dans le choeur de écrivains anciens". De fait, à l'irritation causée par le particularisme religieux des Juifs ou par leur prosélytisme, la cacophonie alexandrine ajoute la notion d'origines impures qui évoque le racisme et celle de crime rituel qui développe la haine populaire et l'autorise à tous les débordements. Le sort ultime de la communauté d'Alexandrie devait montrer que la calomnie peut tuer, surtout lorsqu'elle concerne une minorité que le pouvoir central a des raisons de ne plus défendre. Néanmoins, il ne faut pas oublier quén $\times 115$, ce furent les Juifs qui, pour des raisons encore mal élucidées, prirent l'initiative de se soulever.

Certes, il y a çà et là dans jes écrits grecs ou latins des passages hostiles au judaîsme car il était clair qu'aucun compromis ne pouvait intervenir entre lui et le polythéisme ambiant; symétriquement, il existe une longue tradition biblique de dérision des idoles.

31 Selon Josèphe (Contre Apion II, 79), Apion prend ces fables cheuz deux Syriens, Posidtonios d'Apomee (-135-51) et apollonios Molon (ler siècte). 
R. IItstórla, São Paulo, n. 129-131, p. 141-152, ago.-dez./93 a ago.-dez./94.

Alexandrie est la seule cité où se đéveloppe une littérature anti-juive dont le point culminant paraît se situer au début đu XIer siècle lorsque les Juifs réclament un droit de cité qui leur est contesté: de religieux, le conflit est devenu politique et social. L'historien Tacile importa à Rome les calomnies alexandrines résumées au livre $\mathrm{V}$ de ses Histoires, au lendemain du premier soulèvement de la Judée, donc dans un contexte politique précis; cependant, ces calomnies n'eurent pas d'autre postérité dans la littérature latine. Les Juifs ne constituaient pas une préoccupation centrale dans l'empire paīen. En revanche, its le devinrent dans l'empire chrétien où la haine théologique les diabolisa et produisit une floraison de traites Adversus Judaeos qui accompagnèrent les premières persécutions légales. "L'anti-judaïsme ne devient réellement redoutable qu'avec le développement et le succès du christianisme. L'irrémediable est accompli au sein du Nouveau Testament lui-même (...) De telles formules (...) creusées, approfondies, systématisées par des siècles de meditation théologiques, scelleront le destin des Juifs dans le monde chrétien" ${ }^{32}$.

\section{Bibliographie}

CHeVAluer, Y. L'Antisémitisme. Paris: Cerf, 1988.

GAGER, J.G. The Origins of Antisemitism. Atritudes toward Judaüsm in Pagan and Christian Antiquity. New Yord/Oxford: Oxford University Press, 1983.

HADAS-LEBEL, M.Jérusalem contre Rome. Paris: Cerf, 1990.

MELEZE, J. Les Juifs d'Égypte de Ramses II à Hadrien. Paris: Errance, 1991. NIKIPROWEIZKY, V. De l'antijudaïsme antique a lantisémitisme contemporain. Mélanges d la mémoire de Marcel Préıost. Lille: Presses Universitaires, 1979.

REINACH, Th. Textes d'auteurs grecs et romains relatifs au judaïsme. Paris, 1895.

RUETHER, P. Faith and Fratricide. The Theological Roors of Antisemitism. New York, 1983.

SEVENSTER, N. The Roots of Pagan Antisemitism in the Ancient World. 1975.

STERN, M. Greek and Latin Authors on Jews and JuAaism, vol. I. 1974.

STERN, M. The Jews in Greck and Latin Literature IN: Compendia renum Iudaicarum ad Novum Testamentum, vol. II. Assen, 1976, págs. 1101-1109.

ABSTRACT: Did antisemitism exist in a pagan society before being developed in the heart of Chrislianity? The Jews, whose monotheism isolated them from the environment, constituted a minority

32 NIKIPROWETZKY, op. cir., introctuction. 
HADAS-LEBEL, Mireille. Juits et païens dans le monde grêco-tomain.

frequently threatened amongst the pagans; towever, - and despite their rebellions against Rome - their sight to worship was granted by Roman laws until the beginning of the Christian empire. The image of the Jew illustrated in Greoo-Roman literature since the third century is far from being negative. During the first century, it is necessary to distinguish the exssperalion of the traditional Romans toward the Jewish proselytism from the libel of the Alexandrians which aroused from the ambition of the Jens in obtaining citizenship. Despite the religious conflict, there wasn't the obsession later crented by the tbeological hate.

KEY WORDS: Greco-Roman Antiquity, Judaism, racism, antisemitism, religion. 